\title{
Bibliographie critique sur les Lettres d'un voyageur
}

\section{(2) OpenEdition \\ Journals}

Édition électronique

URL : http://journals.openedition.org/recherchestravaux/184

DOI : 10.4000/recherchestravaux.184

ISSN : 1969-6434

Éditeur

UGA Éditions/Université Grenoble Alpes

\section{Édition imprimée}

Date de publication : 15 avril 2007

Pagination : 193-198

ISBN : 978-2-84310-107-7

ISSN : 0151-1874

Référence électronique

"Bibliographie critique sur les Lettres d'un voyageur », Recherches \& Travaux [En ligne], 70 | 2007, mis en ligne le 28 novembre 2008, consulté le 08 septembre 2020. URL : http://journals.openedition.org/ recherchestravaux/184; DOI : https://doi.org/10.4000/recherchestravaux.184 


\section{BIBLIOGRAPHIE CRITIQUE \\ SUR LES LETTRES D'UN VOYAGEUR}

\section{ÉDITIONS DES LETTRES D'UN VOYAGEUR}

Publication séparée des différentes «Lettres» avant l'édition en recueil (dans la Revue des Deux Mondes, abrégée RDM, pour les onze premières, dans la Revue de Paris pour la douzième):

Lettre I: $R D M, 15$ mai 1834 («Lettres d'un voyageur»).

Lettre II: $R D M, 15$ juillet 1834 («Lettres d'un voyageur, II»).

Lettre III : $R D M$, 15 septembre 1834, («Lettres d'un voyageur, III»).

Lettre IV: $R D M, 1^{\text {er }}$ juin 1836 (début de «Lettres d'un voyageur, VI. Au Malgache»).

Lettre V:RDM, 15 janvier 1835 ("Lettres d'un oncle»).

Lettre VI: RDM, 15 juin 1835 («Lettres d'un voyageur, IV. À Éverard»).

Lettre VII: $R D M, 1^{\text {er }}$ septembre 1835 («Lettres d'un voyageur, V. Sur Lavater et sur une maison déserte. À M. F. Liszt»).

Lettre VIII : RDM, 15 octobre 1834 («Lettre d'un voyageur: le Prince»).

Lettre IX: $R D M, 1^{\text {er }}$ juin 1836 (fin de «Lettres d'un voyageur, VI. Au Malgache»).

Lettre X: RDM, 15 novembre 1836 («Lettres d'un voyageur, VII. À Charles Didier $")$.

Lettre XI : RDM, 15 novembre 1836 («VIII. À Giacomo Meyerbeer»). Lettre XII : Revue de Paris, 29 mai 1836 («Lettre à M. Nisard»).

Lettres d'un voyageur, in Euvres de George Sand, vol. XV et XVI, Paris, Bonnaire, 1837, 2 vol. in- $8^{\circ}$. Lettres d'un voyageur, Bruxelles, Scribe, Tecmen et Cie, 1837, 2 vol. in-18.

Lettres d'un voyageur, in Euvres de George Sand, nouvelle édition revue par l'auteur et accompagnée de morceaux inédits, vol. IX, Paris, Perrotin, 1843, 1 vol. in-18 (apparition de la préface). 
Lettres d'un voyageur, in CEuvres de George Sand, vol. IX, Paris, Garnier frères, 1847,1 vol. in-18.

Lettres d'un voyageur, Paris, Michel Lévy frères, 1857, 1 vol. in-18 (rééditions en 1863 et 1869).

Lettres d'un voyageur (extraits), édition de Pierre Salomon, Paris, Larousse "Classiques Larousse», s. d. (1935 selon le catalogue de la BNF), 1 vol. in-16.

Lettres d'un voyageur, édition de Henri Bonnet, Paris, Flammarion "GF», 1971 (réédité en 2004 avec une bibliographie actualisée).

Lettres d'un voyageur, in Euvres autobiographiques, édition de Georges Lubin, Paris, Gallimard «Bibliothèque de la Pléiade», 1970-1971, 2 vol., vol. II, 1971, p. 633-943.

Lettres d'un voyageur, édition de Suzel Esquier, in Euvres complètes, Béatrice Didier (Dir.), Paris, Honoré Champion (à paraître en 2009).

\section{TraduCtion DES LETTRES D'UN VOYAGEUR À L'ÉTRANGER}

\section{AU XIX ${ }^{\text {e SIECLE }}$}

D'après le recensement établi par Judith Castricum, Corrie Kruikemeier et Suzan van Dijk, "George Sand en traduction", CRIN (Cahiers de recherches des Instituts néerlandais de langue et de littérature françaises), $\mathrm{n}^{\circ}$ 30, 1995, George Sand lue à l'étranger (Recherches nouvelles 3), S. van Dijk (dir.), p. 143-149: en 1835 en russe ( $3^{\mathrm{e}}$ lettre, de 1834); en 1838 en allemand; en 1838 en espagnol; en 1847 en anglais; en 1870 en grec; en 1876 en néerlandais ( $2^{\mathrm{e}}$ lettre, de 1834$)$.

\section{OUVRAGES CRITIQUES COMPORTANT DES ANALYSES SUBSTANTIELLES SUR LES LETTRES D'UN VOYAGEUR}

DIDIER B., George Sand écrivain, "Un grand fleuve d'Amérique ", Paris, PUF « Écrivains", 1998, en particulier p. 291-310.

Doan-Poisson C. T., Poétique de la mobilité. Les lieux dans Histoire de ma vie de George Sand, Amsterdam-Atlanta, Rodopi, 2000, passim.

Hoog-Naginski I., George Sand, l'écriture ou la vie, Paris, H. Champion, 1999, passim.

LAFORGUE P., Romanticoco. Fantaisie, chimère et mélancolie (1830-1860), Saint-Denis, Presses universitaires de Vincennes "L'Imaginaire du Texte ", 2001, p. 109-118.

-, Corambé. Identité et fiction de soi chez George Sand, Paris, Klincksieck, 2003, en particulier p. 19-26 et p. 75-95. 
MariX-Spire Th., Les Romantiques et la musique. Le cas George Sand, 18041838, Paris, Nouvelles Éditions latines, 1954, passim.

McCall Saint-Saens A. E., De l'être en lettres. L'autobiographie épistolaire de George Sand, Amsterdam-Atlanta, Rodopi, 1996, passim.

Mozet N., George Sand, écrivain de romans, Saint-Cyr-sur-Loire, Christian Pirot éditeur, 1997, en particulier p. 27-53.

POWELl D. A., While the Music Lasts. The Representation of Music in the works of George Sand, Londres, Associate University Presses, 2001, en particulier p. 255-261.

Vierne S., George Sand, la femme qui écrivait la nuit, Clermont-Ferrand, Presses universitaires Blaise-Pascal "Cahiers romantiques, $n^{\circ}$ 9», 2004, passim.

ARTICles CONSACRÉS, POUR TOUT OU PARTIE, AUX LETTRES D'UN VOYAGEUR

BEDNER J., "Cet amour imprimé” : les Lettres d'un voyageur et La Confession d'un enfant du siècle», (En)jeux de la communication romanesque, S. van Dijk et Ch. Stevens (dir.), Amsterdam-Atlanta, Rodopi, 1994, p. 85-95.

BERNARD-GriffithS S., "Venise palimpseste: des Lettres d'un voyageur (1834) à Mattea (1835) de George Sand", Présences de l'Italie dans l'ceuvre de George Sand, Moncalieri (Italie), Centre interuniversitaire de recherche sur le voyage en Italie «Bibliothèque du voyage en Italie», Études n ${ }^{\circ} 66$, 2004, p. 387-409.

BERTHIER, Ph., "Lettres d'un voyageur: le cru et le cuit", Ville, campagne et nature dans l'ceuvre de George Sand, S. Bernard-Griffiths (dir.), ClermontFerrand, Presses universitaires Blaise-Pascal «Révolutions et Romantismes», 2002, p. 135-147.

BOCHENEK-FRANCZAKOWA R., "Les Lettres d'un voyageur: jeux et enjeux de la communication épistolaire", Les Amis de George Sand, n 24, 2002, p. 31-39.

Bonnet H., "George Sand et Gérard de Nerval», Hommage à George Sand, L. Cellier (dir.), Paris, PUF, 1969, p. 115-150.

—, "Le "bouquet" sandien. Des Lettres d'un voyageur aux Nouvelles Lettres d'un voyageur", Fleurs et jardins dans l'ouvre de George Sand, S. BernardGriffiths et M.-C. Levet (dir.), Clermont-Ferrand, Presses universitaires Blaise-Pascal «Révolutions et Romantismes», 2006, p. 371-392.

Bozon-SCALZITTI Y., «La pierre, l'eau, le sable: l'écriture sandienne dans les Lettres d'un voyageur", Nineteenth-Century French Studies, vol. XXI, n 34, 1993, p. 339-356. 
Chovelon B., "George Sand, voyageuse dans les Alpes", Études françaises, vol. 24, n 1, printemps 1988, George Sand. Voyage et écriture, J. Goldin (dir.), p. 17-27.

CZYBA L., «La représentation de Venise dans les "Lettres d'un voyageur, II et III" ", George Sand et son temps. Hommage à Annarosa Poli, E. Mosele (dir.), Genève, Slatkine, 1994, 3 vol., vol. II, p. 789-807.

DALE C., "The mirror of romanticism. Images of music, religion and art criticism in Sand's eleventh Lettre d'un voyageur to Giacomo Meyerbeer", The Romanic Review, vol. 87 n 1, janv. 1996, p. 83-112.

DiAZ B., "Les Lettres d'un voyageur de George Sand: un voyage esthétique», L'Esthétique en acte. Actes du II' Congrès de la Société des études romantiques et dix-neuviémistes, 26-28 mai 2005, à paraître en 2007.

DiaZ J.-L., "Opéra par lettres: "L'aventure de Venise" selon les deux premières "Lettres d'un voyageur", Présences de l'Italie dans l'auvre de George Sand, Moncalieri (Italie), Centre interuniversitaire de recherche sur le voyage en Italie "Bibliothèque du voyage en Italie», Études n 66, 2004, p. 411-424.

—, «Entre l'art et la politique: le moment des Lettres d'un voyageur (18341837)", Les Héritages de George Sand aux XXe et XXIe siècles. Les arts et la politique. Études réunies et présentées par la Société japonaise des études sandiennes, H. Nishio (dir.), Tokyo, Keio University Press, 2006, p. 191207.

DiDiER B., «La musique italienne dans les Lettres d'un voyageur», Présences de l'Italie dans l'ouvre de George Sand, Moncalieri (Italie), Centre interuniversitaire de recherche sur le voyage en Italie «Bibliothèque du voyage en Italie», Études n 66, 2004, p. 149-160.

GARNETT M. A., "Imaginary and symbolic orders in "Sur Lavater et sur une maison déserte" ", The World of George Sand, N. Datlof, J. Fuchs et D.A. Powell (dir.), New York - Westport - Londres, Greenwood Press, 1991, p. 45-50.

—, "A pilgrim's progress. Biblical metaphors in the Lettres d'un voyageur», The Traveler in the life of George Sand, T. Alvarez-Detrell et M.G. Paulson (dir.), Troy (NY), Whitston, 1993, p. 41-53.

Gaudon J., "Sand et Hugo, voyageurs», George Sand et son temps. Hommage à Annarosa Poli, E. Mosele (dir.), Genève, Slatkine, 1994, 3 vol., vol. III, p. 1417-1428.

GENEVRAY F., «Le motif tyrolien dans la première "Lettre d'un voyageur" de Sand», Studi Francesi, n 117, XXXIX e année, sept.-déc. 1995, p. 496502. 
—, "Des Lettres d'un voyageur à Teverino: George Sand et le voyageur sans bagage", Romantisme, ${ }^{\circ}$ 99, $1^{\text {er }}$ trim. 1998, p. 39-51.

—, "Bohême, bohème, bohémien : autour de George Sand ", exposé présenté lors du séminaire de formation doctorale «L'auteur en marge», 10 avril 2003, consultable sur Internet: www.univ-lyon3.fr (Recherche/Ressources/Articles).

-, "Réminiscences antiques dans les Lettres d'un voyageur", Les Amis de George Sand, n² 27, 2005, p. 23-37.

—, "Adresse épistolaire et écrits intimes: manipulations intergénériques autour des Lettres d'un voyageur", George Sand. Pratiques et imaginaires de l'écriture. Actes du colloque de Cerisy-la-Salle, 1-8 juillet 2004, B. Diaz et I.H. Naginski (dir.), Caen, Presses universitaires de Caen, 2006, p. 5969.

—, "Les Lettres d'un voyageur relues par Histoire de ma vie: un double pacte ", George Sand Studies, D. Laporte (dir.), vol. XXV, 2006, p. 32-51.

—, "Lorsque George Sand se relit", La Relecture de l'ceuvre par ses écrivains mêmes, M. Hilsum (dir.), Paris, Kimé "Cahiers de Marge», 2007, 2 vol., vol. I, p. 187-199.

Goldin J., "Voyage et descriptif sandien: une série infinie de miroirs", George Sand Studies, F. Massardier-Kenney (dir.), vol. XXII, 1993, p. 210.

Gorilovics T., «Un regard d'homme: le narrateur sandien», George Sand. L'Écriture du roman, J. Goldin (dir.), Montréal, université de Montréal «Paragraphes, $n^{\circ} 12 », 1996$, p. 251-259.

HaRLAN E., "Lettres d'un voyageur, or going home again", The Traveler in the life of George Sand, T. Alvarez-Detrell et M. G. Paulson (dir.), Troy (NY), Whitston, 1993, p. 16-29.

Hoog M.-J.: «Lettres d'un voyageur, texte initiatique», George Sand. Actes du colloque de Cerisy-la-Salle, 13-23 juillet 1981, Simone Vierne (dir.), Paris, Sedes, 1983, p. 137-147.

LÉGER S. H., "Lettres d'un voyageur. Traveling with Sand», The World of George Sand, N. Datlof, J. Fuchs et D. A. Powell (dir.), New YorkWestport-Londres, Greenwood Press, 1991, p. 51-56.

Le Huenen R., "George Sand et l'écriture du voyage: à propos des Lettres d'un voyageur", George Sand. George Sand. Pratiques et imaginaires de l'écriture. Actes du colloque de Cerisy-la-Salle, 1-8 juillet 2004, B. Diaz et I.H. Naginski (dir.), Caen, Presses universitaires de Caen, 2006, p. 203214. 
McCall Saint-Saens A. E., "On the heels of Corinne: Venice, Sand's traveler and a case for urban renewal (George Sand, Lettres d'un voyageur)", The Romanic Review, vol. 89 n 2, mars 1998, p. 219-230.

—, "Au delà du sale et du propre: des Lettres d'un voyageur à La Daniella», Présences de l'Italie dans l'ouvre de George Sand, Moncalieri (Italie), Centre interuniversitaire de recherche sur le voyage en Italie "Bibliothèque du voyage en Italie», Études n ${ }^{\circ} 66,2004$, p. 307-322.

Monicat B., "Les Lettres d'un voyageur: récit de voyage au féminin?», George Sand Studies, F. Massardier-Kenney (dir.), vol. XII, 1993, p. 1117.

Mozet N., «Du bon usage (littéraire) de la lettre de rupture: du Lys dans la vallée aux Lettres d'un voyageur", Revue des Sciences humaines, $\mathrm{n}^{\circ} 195$, juil.-sept. 1984, p. 19-24.

—, "Signé "le voyageur": George Sand et l'invention de l'artiste», Romantisme, $\mathrm{n}^{\circ} 55,1^{\mathrm{er}}$ trim. 1987, p. 23-32.

—, "Le voyageur sandien en quête d'un lieu d'écriture», Études françaises, vol. 24, n 1, printemps 1988, George Sand. Voyage et écriture, J. Goldin (dir.), p. 41-55.

Noland A., "Nietzsche on Sand: a note", George Sand Studies, vol. IX, 1988-1989, p. 48-52.

POLI A., "Il viaggio reale e il viaggio immaginario nelle Lettres d'un voyageur de George Sand", La Letteratura e l'Immaginario. Problemi di sementica e di storia del lessico franco-italiano, G. Cesbron (dir.), Milan, CisalpinoGoliardica, 1984, p. 80-91.

—, "Lamennais à l'île San Lazzaro, voyage réel ou voyage imaginaire?», Voyage imaginaire, voyage initiatique, Moncalieri-Genève, Cirvi-Slatkine "Dimension du voyage», 1989, p. 45-69.

Ragni S., "Les Huguenots di Meyerbeer, tra George Sand e Mazzini", Bollettino della Domus mazziniana, vol. 39, n 1, 1993, p. 161-177.

Rogers, Nancy E.: "Modes of travel in Sand's Lettres d'un voyageur", The Traveler in the life of George Sand, T. Alvarez-Detrell et M. G. Paulson (dir.), Troy (NY), Whitston, 1993, p. 30-40.

SCHAeffer G., "George Sand voyageuse», Romantisme, n 4, 1972, p. 100111.

SZMURLo K., "Géographie de femme dans les Lettres d'un voyageur», George Sand Studies, A. E. McCall (dir.), vol. XVI, 1997, p. 17-27.

VIERne S., "George Sand: la halte et le chemin", George Sand Studies, F. Massardier-Kenney (dir.), vol. XII, 1993, p. 26-35. 